\title{
Comparison of Cardiovascular, Analgesic and Sedative Effects of Different Doses of Dezocine
}

\author{
Chang-Ming Wang1, Zhan-Ying Guan², Chang-Hua Cai¹, Jing \\ Zhang1, Quan Cai Wang ${ }^{3}$, Yi Ma $^{3}$ and Ping Zhao ${ }^{4 *}$ \\ 1Department of Anesthesiology, The People's Hospital of China Medical University, \\ China
}

2Department of Anesthesiology, Branch Hospital of Hunnan, China

\section{Research Article \\ Volume 2 Issue 1}

Received Date: July 07, 2017

Published Date: July 18, 2017

3Department of Neurosurgery, The People's Hospital of China Medical University, China

${ }^{4}$ Department of Anaesthesiology, Shengjing Hospital of China Medical University, China

*Corresponding author: Ping Zhao, Department of Anaesthesiology, Shengjing Hospital of China Medical University, No 36, San hao Street, Herping District, Shenyang 110004, China, Tel: 024-24016452; E-mail: zhaopingdoctor@yeah.net

\section{Abstract}

Background: Dezocine is considered to be an alternative medication for managing postoperative pain. However, no study is focused on the cardiovascular, analgesic and sedative effects of intravenous Dezocine during anesthesia recovery period after micro vascular decompression (MVD) surgery for hemi facial spasm (HS).Therefore; we provide the clinical practice on this topic.

Methods: A total of 160 patients, American Society of Anesthesiologists (ASA) physical status I-II, undergoing elective MVD surgery for HS, were randomized equally into four groups. The four groups were given intravenously a matching placebo (group A) (equal volume of $0.9 \%$ saline), Dezocine $0.05 \mathrm{mg} / \mathrm{kg}$ (group B), $0.1 \mathrm{mg} / \mathrm{kg}$ (group C) and $0.2 \mathrm{mg} / \mathrm{kg}$ (group D) respectively. Mean arterial pressure (MAP) and heart rate (HR) were measured at three periods: 5minutes before anesthesia induction (T0), at the moment of pulling out laryngeal mask (LMA) (T1),10 minutes after the procedure(T2).Sedation-Agitation Scale (SAS) at T1, Visual Analogue Scale (VAS) and Modified Observers Assessment of Alertness/Sedation Scale (OAA/S)at T2 were assessed in four groups. Mean while, the incidences of respiratory depression (RD), postoperative nausea and vomiting (PONV) were also observed during anesthesia recovery period in four groups. Multivariate analysis of variance and the Pearson $\chi 2$ test were used, and a value of $\mathrm{P}<0.05$ was considered statistically significant.

Results: The mean value of anesthesia recovery time was higher in group $\mathrm{D}(\mathrm{P}<0.05$ vs. group A).It indicated statistical differences $(\mathrm{P}<0.05 \mathrm{vs}$. group $\mathrm{A})$ in $\mathrm{SAS}$ and VAS at $\mathrm{T} 1$ in group $\mathrm{C}$ and in group $\mathrm{D}$, the value of $0 \mathrm{AA} / \mathrm{S}$ indicated statistical difference $(\mathrm{P}<0.05 \mathrm{vs}$. group $\mathrm{A})$ at $\mathrm{T} 2$ in group $\mathrm{D}$. It indicated significant differences $(\mathrm{P}<0.05$ vs. group $\mathrm{A})$ in MAPand $\mathrm{HR}$ at 


\section{Anaesthesia \& Critical Care Medicine Journal}

T1 andT2 in group C and group D. The occurrence of PONV was 2.5\% (group A),2.5\% (group B),5.0\% ( group C) and $12.5 \%$ ( group D) separately. No patient in the four groups had RD.

Conclusions: The results of current study suggests that Dezocine can effectively prevent the abrupt rise of blood pressure and HR, enhance analgesia and sedation without RD during anesthesia recovery period after MVD surgery for HS. However, Dezocine is dose-dependent in prolonging anesthesia recovery time and increasing he incidence of PONV.

Keywords: Dezocine; Cardiovascular; Analgesic; Sedative

\section{Introduction}

Dezocine, as an opioidmu receptor partial agonist/antagonist, is considered to be an alternative medication for managing postoperative pain. Common adverse effects observed in opioids with full agonism are significantly reduced due to its partial agonism on the mu opioid receptor [1-4]. Similar to other opioids, Dezocine could decrease anesthetic requirement by up to $50 \%$ [5]. Because of above-mentioned reasons, although no longer used clinically in Western countries, Dezocine has been used widely in China as an alternative medication for postoperative pain management [3]. Micro vascular decompression (MVD) surgery has been regarded widely as a positive effective treatment method for hemi facial spasm (HFS)[6-8]. However, MVD does have potential risks of complications resulting from retromastoidsuboccipital craniectomysuch as postoperative headache and hemorrhage $[8,9]$. Acute pain, due to surgical tissue and nerve trauma, can lead to significant hemodynamic changes and even serious complications during anesthesia recovery period, which needs to be paid close attention to. To our knowledge, whether clinically administrated Dezocine can provide a more satisfying cardiovascular, analgesic and sedative effects during anesthesia recovery period have never been documented. Therefore, we designed the trial to investigate the cardiovascular, analgesic and sedative effects of different doses of Dezocine during anesthesia recovery after MVD surgery for HFS.

\section{Patients}

Ethics approval for this study was provided by the Institutional Ethics Committee (Liaoning Provincial People's Hospital) on March 1, 2013. After obtaining the approval and informed consent, 160 ASA I-II patient switcher-Pharyngeal Airway Cap(OPLAC, XU BANG Corporation, Taiwan, China) laryngeal mask (LMA) anesthesia included in this study(C.-M.W. took part in operations, which were included in the study, others were excluded), aged 24 to 78 years and weighed 45 to 97kgwith HFS who were scheduled to receive MVD, between March 2013 and December 2016, were randomly divided equally into four groups (each group, $\mathrm{n}=40$ ). The four groups were given intravenously a matching placebo (group A) (equal volume of $0.9 \%$ saline), Dezocine (Yangtze River Pharmaceutical Group, Taizhou, Jiangsu, China) $0.05 \mathrm{mg} / \mathrm{kg}$ (group B), $0.1 \mathrm{mg} / \mathrm{kg}$ (group C) and $0.2 \mathrm{mg} / \mathrm{kg}$ (group D)respectively. No patient showed neurological abnormalities before the start of anesthesia.

\section{Anesthetic Procedures}

All patients were told to prohibit food and water for no less than 8 hours before anesthesia. Every patient was given intramuscular injection atropine $0.5 \mathrm{mg} 30$ minutes before operation (all patients had no contraindication in atropine application, such as glaucoma). After patients entered into the operating room, peripheral intravenous cannula was placed. Meanwhile, blood pressure (BP), electrocardiogram (ECG) and pulse oxygen saturation (Sp02) were monitored throughout the whole procedure. Anesthesia was induced using propofol $2.0 \mathrm{mg} / \mathrm{kg}$ over 20 seconds followed immediately by fentanyl $4 \mu \mathrm{g} / \mathrm{kg}$; intravenous vecuronium $0.1 \mathrm{mg} / \mathrm{kg}$ was administered 20seconds after completing a bolus fentanyl. As soon as the patients lost consciousness (unconsciousness was measured by eyelash reflex), ventilation was attempted using a mask. Subsequently a properly sizedOPLAClaryngeal mask was inserted by using index finger insertion method within 60seconds, then patients were placed in the park bench position (Significantly, if OPLAC ventilation was not good on the supine position, OPLACLMA should be timely replaced with tracheal intubation). Anesthesia was maintained using

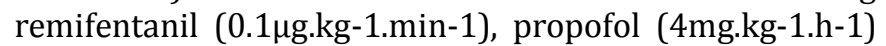
continuous pump infusion and inhalation of $1.5 \%$ sevoflurane to maintain the depth of anesthesia. In 


\section{Anaesthesia \& Critical Care Medicine Journal}

addition, half the initial dose of vecuronium was appended at the beginning of microscope operation and saline or Dezocine was injected intravenously at the moment of microscopy removed. Sevoflurane was stopped after the dura closed. Remifentanil and propofol were stopped before starting to close the skin. When patients' consciousness, muscle tension, autonomousrespirationand reflex restored, OPLACLMA was extubated and patients were returned to the wards after being observed for 10 minutes.

\section{Data Recording and Clinical Evaluation}

Mean arterial pressure (MAP) and heart rate (HR) were measured at three periods: 5 minutes before anesthesia induction (T0), at the moment of pulling out LMA (T1), 10 minutes after the procedure (T2). The time, from discontinuation of propofol and remifentanil to patients called opening eyes, was called anesthesia recovery time. Reference standard for sedation and analgesia during anesthesia recovery: The visual Analogue Scale (VAS; from 0 to 10; 0 , no pain; 10, worst pain imaginable), Sedation-Agitation Scale (SAS) (Table 1) and Modified Observers Assessment of Alertness/Sedation Scale (OAA/S) (Table 2). SAS at T1, VAS and OAA/Sat T2 were assessed. Postoperative nausea and vomiting (PONV) and respiratory depression (RD) were also evaluated during anesthesia recovery in four groups. Criteria for definitive RD were as follows: (1) patient received nalox one and indicated evidence of reversal of RD,(2) other clear and objective signs of RD or opioid toxicity, for example, patient with the clinical signs such as over sedation, respiratory arrest, and need intervention $[10,11]$.

\begin{tabular}{|c|c|c|}
\hline Score & Term & Description \\
\hline 7 & Dangerous agitation & $\begin{array}{l}\text { Pulling at endotracheal tube, trying to remove catheters, climbing over bed rail, } \\
\text { striking at staff, thrashing side to side }\end{array}$ \\
\hline 6 & Very agitated & $\begin{array}{c}\text { Does not calm, despite frequent verbal reminding of limits; requires physical } \\
\text { restraints, biting endotracheal tube }\end{array}$ \\
\hline 5 & Agitated & Anxious or mildly agitated, attempting to sit up, calms down to verbal instructions \\
\hline 4 & $\begin{array}{l}\text { Calm and } \\
\text { cooperative }\end{array}$ & Calm, awakens easily, follows commands \\
\hline 3 & Sedated & $\begin{array}{l}\begin{array}{l}\text { Difficult to arouse; awakens to verbal stimuli or gentle shaking, but drifts off again; } \\
\text { follows simple commands }\end{array} \\
\end{array}$ \\
\hline 2 & Very sedated & $\begin{array}{l}\text { Arouses to physical stimuli, but does not communicate or follow commands, may } \\
\text { move spontaneously }\end{array}$ \\
\hline 1 & Unable to rouse & $\begin{array}{l}\text { Minimal or no response to noxious stimuli, does not communicate or follow } \\
\text { commands }\end{array}$ \\
\hline
\end{tabular}

Table 1: The Sedation-Agitation Scale.

\begin{tabular}{|c|c|}
\hline Response & Score level \\
\hline Responds readily to their name spoken in a normal tone & 5 (Alert) \\
\hline Lethargic response to their name spoken in a normal tone & 4 \\
\hline Response only after their name is called loudly and/or repeatedly & 3 \\
\hline Response only after name spoken with mild prodding or shaking & 2 \\
\hline Does not respond to mild prodding or shaking & 1 \\
\hline Does not respond to noxious stimuli (Trapezius squeezing) & 0 \\
\hline
\end{tabular}

Table 2: Responsiveness Scores of the Modified Observer 's Assessment of Alertness/Sedation Scale( OAA/S). 


\section{Anaesthesia \& Critical Care Medicine Journal}

\section{Statistical Analysis}

Numerical data was all reported in mean? SD (mean of standard deviations). Variables were tested for normal distribution using the Kolmogorov-Smirnov test and Q-Q Pearson $\chi 2$ test. $\mathrm{P}<0.05$ was considered to be statistical difference.

\section{Results}

A total of 160 patients were recruited into this study. As shown in Table 3, there were no substantive betweengroup differences in the age, sex, diseased region, operation time and weights. Compared with group A, group D indicated statistically significant difference in anesthesia recovery time (group D, group A: $9.35 \pm 1.98$, $6.40 \pm 1.25 \mathrm{~min}, \mathrm{P}<0.05 \mathrm{vs}$. group $\mathrm{A}$ ), no significant difference was found in other groups. As shown in Figure plots. The parametric test of variance analysis for clinical parameters in the above-mentioned subgroups of patients was assessed using the multivariate analysis of variance (MANOVA). Demographic data were compared using the

1, compared with group A, the mean values of MAP at T1 and $\mathrm{T} 2$ were lower in group $\mathrm{C}$ (group $\mathrm{C}$, group $\mathrm{A}: \mathrm{T} 1$, $95.05 \pm 5.79, \quad 106.75 \pm 7.40 \mathrm{mmHg} ; \quad \mathrm{T} 2, \quad 93.25 \pm 4.79$, $102.4 \pm 6.35 \mathrm{mmHg}, \mathrm{P}<0.05$ vs. group A) and group D (group D, group A: T1, 94.10 $\pm 6.14,106.75 \pm 7.40 \mathrm{mmHg}$; T2, 93.03 $\pm 5.82,102.4 \pm 6.35 \mathrm{mmHg}, \mathrm{P}<0.05$ vs. group A). Meanwhile, the mean values of HR at T1 andT2were also lower in group C (group C, group A: T1, 83.90 \pm 5.88 , $87.03 \pm 7.86 \mathrm{bpm}$; T2, 80.65 $\pm 5.25,84.8 \pm 7.58 \mathrm{bpm}, \mathrm{P}<0.05$ vs. group A) and group D (group D, group A: T1, 82.60 $\pm 7.19, \quad 87.03 \pm 7.86 \mathrm{bpm} ; \quad \mathrm{T} 2, \quad 80.28 \pm 6.17$, $84.8 \pm 7.58 \mathrm{bpm}, \mathrm{P}<0.05$ vs. group $\mathrm{A})$.

\begin{tabular}{|c|c|c|c|c|c|}
\hline & $\begin{array}{c}\text { group } A \\
(n=40)\end{array}$ & $\begin{array}{c}\text { group B } \\
(n=40)\end{array}$ & $\begin{array}{c}\text { group C } \\
(n=40)\end{array}$ & $\begin{array}{c}\text { group D } \\
(n=40)\end{array}$ & $P$-value \\
\hline \multicolumn{6}{|l|}{ Sex } \\
\hline Male & 8 & 10 & 11 & 10 & \multirow{2}{*}{0.866} \\
\hline Female & 32 & 30 & 29 & 30 & \\
\hline \multicolumn{6}{|l|}{ Diseased region } \\
\hline Left & 25 & 22 & 22 & 24 & \multirow{2}{*}{0.875} \\
\hline Right & 15 & 18 & 18 & 16 & \\
\hline Mean age $(\mathrm{y})$ & $52.90 \pm 11.50$ & $52.72 \pm 8.69$ & $52.24 \pm 8.32$ & $52.37 \pm 10.59$ & 0.069 \\
\hline $\begin{array}{c}\text { Mean weight } \\
(\mathrm{kg})\end{array}$ & $62.34 \pm 6.85$ & $62.19 \pm 7.48$ & $62.52 \pm 8.42$ & $62.50 \pm 6.55$ & 0.370 \\
\hline $\begin{array}{l}\text { Anesthesia } \\
\text { recovery time } \\
\text { (min) }\end{array}$ & $111.39 \pm 10.15$ & $111.71 \pm 8.74$ & $111.51 \pm 11.07$ & $111.64 \pm 10.52$ & 0.701 \\
\hline $\begin{array}{l}\text { Operation time } \\
\text { (min) }\end{array}$ & $6.40 \pm 1.25$ & $6.48 \pm 1.72$ & $6.39 \pm 1.64$ & $9.35 \pm 1.98^{\#}$ & 0.000 \\
\hline
\end{tabular}

Table 3: Demographic Data of group A, group B, group C and group D ( $\bar{x} \pm \mathrm{s}, \mathrm{n})$.

${ }^{\#} P<0.05$, There were statistical differences between group $B$, group $C$ and group $D$ vs. group $A$. 


\section{Anaesthesia \& Critical Care Medicine Journal}

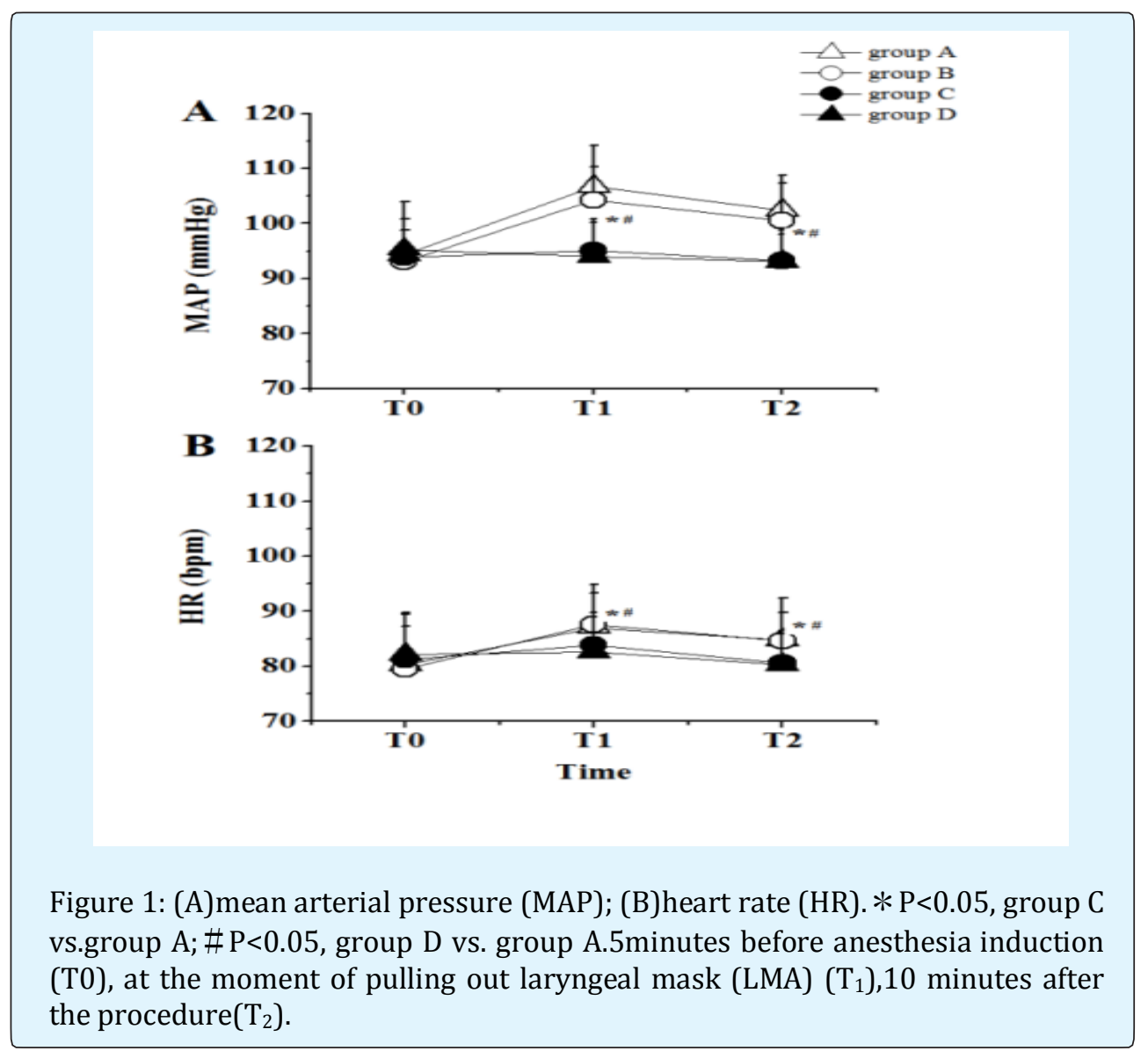

Means of SASindicated statistical differences at T1 in group C (group C, group A: $4.48 \pm 0.78,5.13 \pm 1.11$ points, $\mathrm{P}<0.05$ vs. group $\mathrm{A}$ ) and in group $\mathrm{D}$ (group $\mathrm{D}$, group A:4.00 $\pm 0.78,5.13 \pm 1.11$ points, $P<0.05$ vs. group A); Means of VAS indicated statistically significant differences at T2 in group C (group C, group A: $1.17 \pm 0.71,3.35 \pm 1.14$ points, $\mathrm{P}<0.05$ vs. group $\mathrm{A}$ ) and group $\mathrm{D}$ (group $\mathrm{D}$, group $\mathrm{A}$ :
$1.05 \pm 0.81,3.35 \pm 1.14$ points, $\mathrm{P}<0.05$ vs. group A); Means of $\mathrm{OAA} / \mathrm{S}$ was lower at T2 in group D (group D, group A: $4.15 \pm 0.83,4.58 \pm 0.55$ points, $\mathrm{P}<0.05$,vs. group A) (Table 4). The occurrence of PONV in four groups was $2.5 \%$ ( 1 of 40 ,group A), $2.5 \%$ (1 of 40,group B), $5.0 \%$ (2of 40, group C) and $12.5 \%$ (5 of 40 , group D) separately. Howbeit none of the patients suffered from RD in the four groups.

\begin{tabular}{|l|l|l|l|l|}
\hline & Cases(n) & $\mathbf{T}_{1}($ SAS, point) & $\mathbf{T}_{2}$ (VAS,point ) & $\mathbf{T}_{2}(\mathbf{O A A} / \mathbf{S}$, point) \\
\hline group A & 40 & $5.13 \pm 1.11$ & $3.35 \pm 1.14$ & $4.58 \pm 0.55$ \\
\hline group B & 40 & $5.05 \pm 1.06$ & $3.15 \pm 1.21$ & $4.60 \pm 0.50$ \\
\hline group C & 40 & $4.48 \pm 0.78^{\#}$ & $1.17 \pm 0.71^{\#}$ & $4.40 \pm 0.63$ \\
\hline group D & 40 & $4.00 \pm 0.78^{\#}$ & $1.05 \pm 0.81^{\#}$ & $4.15 \pm 0.83^{\#}$ \\
\hline
\end{tabular}

Table 4: Means of SAS at $\mathrm{T}_{1}, \mathrm{VAS}$ and OAA/S at $\mathrm{T}_{2}$ in group $\mathrm{A}$, group $\mathrm{B}$, group $\mathrm{C}$ and group $\mathrm{D}(\bar{x} \pm \mathrm{s}, \mathrm{n})$.

${ }^{\#} P<0.05$,There were statistical differences between group $\mathrm{B}$, group $\mathrm{C}$ and group $\mathrm{D}$ vs. group $\mathrm{A}$.

\section{Discussion}

In the clinical practice, compared with the placebo, $0.05 \mathrm{mg} / \mathrm{kg}$ Dezocine did not show significant difference in all respects observed, it meant that $0.05 \mathrm{mg} / \mathrm{kg}$ Dezocine given intravenously was ineffective in analgesia and sedation. In contrast, whether $0.1 \mathrm{mg} / \mathrm{kg}$ dezocine or $0.2 \mathrm{mg} / \mathrm{kg}$ Dezocine given intravenously was effective in preventing signific anthemo dynamic changes, analgesia and sedation. There was no difference in the incidence of 


\section{Anaesthesia \& Critical Care Medicine Journal}

PONV following $0.05 \mathrm{mg} / \mathrm{kg}$ dezocine and $0.1 \mathrm{mg} / \mathrm{kg}$ Dezocine given intravenously treatment compared with the placebo. However, $0.2 \mathrm{mg} / \mathrm{kg}$ Dezocine given intravenously may delay postoperative recovery time and increase the incidence of PONV. This study provided clinical experimental evidence that dezocine dose dependently increased the incidence of PONV. These results indicated that $0.1 \mathrm{mg} / \mathrm{kg}$ Dezocine given intravenously can take on the roles for relieving postoperative pain and reducing the incidence of emergence agitation with less affecting the postoperative recovery time. To the best of our knowledge, this is the first study to assess the cardiovascular, analgesic and sedative effect of intravenous dezocine during anesthesia recovery period after MVD surgery for HS. Furthermore, few studies are focused on dezocine-related complications, such as RD and PONV.

A recent study conducted in a cohort of adult surgical patients in the United States reveals that 86\% experience pain after surgery, of which $75 \%$ have moderate-tosevere pain in the immediate postoperative period [12]. Currently, despite the increasing use of a variety of different analgesic strategies, opioids continue as the mainstay for management of moderate to severe acute pain after surgery $[13,14]$, their analgesic effects are offset by undesired adverse effects, including RD, drug addiction, and PONV [15-17]. What merits our primary concern is that opioids inducing PONV and RD is the most common side effect for acute postoperative pain. Therefore, the accompanying adverse effects of opioids are not negligible and inevitably limit its clinical use. The ideal postoperative pain management should relieve pain and keeping adverse effects to a minimum [18]. Dezocine, first developed in 1970s, is an opioid murecept or partial agonist/antagonist [1]. Theoretically; it could antagonize the anti-nociceptive effects of morphine. Nevertheless, Dezocine concentration-dependently enhances the analgesic effects of morphine [19]. It suggests the existence of alternative targets for Dezocine that could have additive effects for opioids. Based on the above reasons, some common adverse effects of opioids are significantly reduced with this drug due to its good tolerance, mild adverse effects and good potence $[1,20$ 22]. Some studies find that Dezocine is a promising and safe analgesic that is slightly more potent than morphine for the relief of postoperative pain [1,23]. Most importantly, study indicates that Dezocine can cause a significant increase in the number of patients with at least $50 \%$ pain relief during the early postoperative period (within 0-1h) compared with morphine [1], which means that Dezocine may be a good choice for postoperative pain during anesthesia recovery period. Therefore, dezocine is gaining popularity in China as an alternative for postoperative pain [3]. Postoperative pain is one of many factors attributing to emergence agitation which is common that occurs during the early stage of recovery from general anesthesia [18]. Nevertheless, postoperative pain and emergence agitation may lead to serious consequences, such as significant hemodynamic changes, removal of catheters, self-extubation, hemorrhage, and even severe injuries from falling out of the bed. Furthermore, it may increase the demand on human resources and even lead to medical staff injuries.

MVD surgery has been identified as a well-accepted and positive effective treatment method for medicationintractable HFS and has proved to be valuable to cure this disease [8,24-28]. However, MVD does have potential risks of complications, such as postoperative headache and hemorrhage [29-31]. Accordingly, it is necessary for controlling postoperative pain during anesthesia recovery after MVD surgery for HFS. Acute postoperative pain, especially in anesthesia recovery after MVD surgery for HFS, which is a normal response to surgical intervention, can lead to pain-related complications, such as cardiovascular complications. Thus far, there is few study to focus on cardiovascular, analgesic and sedative effects of dezocine during anesthesia recovery period after MVD surgery for HFS [32]. Several limitations inherent of the present study should be highlighted. Firstly, the number of small-scale trials limits the accuracy in complications observation during anesthesia recovery period. Large studies will be necessary to adequately evaluate the adverse effects of this drug. Although no patient has RD in this study, the current dose of medicine may be insufficient to lead to RD. Because of Dezocine partial muagonism, it should exhibit a "ceiling effect" for RD. Secondly, this study provides some references for clinical treatment but cannot provide the optimal dose of dezocine for preventive analgesia due to individual differences in patients, degree of surgical trauma, the time points of drug application and reasonable concentration gradient. Here, we only observe anaesthesia with OPLACLMA which has less stimulation for respiratory tract more than anaesthesia with intubation during anesthesia recovery period. Compared with anaesthesia with OPLACLMA, the results may be different in anaesthesia with intubation. Thirdly, this study is lack of relatively objective indicators of sedation monitoring, such as bispectral index (BIS) monitoring. In addition, MVD surgery for HFS anaesthesia-related studies is less, especially for anesthesia recovery period. It means that less experience is got for us from previous studies. 


\section{Anaesthesia \& Critical Care Medicine Journal}

\section{Conclusion}

In conclusion, this study demonstrated that the administration of dezocine was indeed effective in preventing significant hemodynamic changes, enhancing postoperative analgesia and sedation during anesthesia recovery period after MVD surgery for HFS. With the increase of dosage, dezocine can prolong anesthesia recovery time and increase the incidence of PONV. However, our results did not discover the occurrence of RD. Further studies are warranted.

\section{Conflict of Interests}

None of the authors has any conflicts of interest.

\section{Funding}

This work was funded by Liao ning Natural Science Foundation of China (No. 20170540524).

\section{References}

1. Liu R, Huang XP, Yeliseev A, Xi J, Roth BL (2014) Novel molecular targets of dezocine and their clinical implications. Anesthesiology 120(3): 714-723.

2. Wang X, Thirumala PD, Shah A, Gardner P, Habeych $M$, et al. (2013)Microvascular decompression for hemifacial spasm: focus on late reoperation. Neurosurg Rev 36(4): 637-644.

3. Zhou X, Zhang C, Wang M, Yu L, Yan M (2015) Dezocine for Preventing Postoperative Pain: A MetaAnalysisof Randomized Controlled Trials. PLoS One 10(8): e0136091.

4. Jacobs AM, Youngblood F (1992) Opioid receptor affinity for agonist-antagonist analgesics. J Am Podiatr Med Assoc 82(10): 520-524.

5. Rowlingson JC, Moscicki JC, DiFazio CA (1983) Anesthetic potency of dezocine and its interaction with morphine in rats. Anesth Analg 62: 899-902.

6. Joo BE, Park SK, Cho KR, Kong DS, Seo DW, et al. (2016) Real-time intraoperative monitoring of brainstem auditory evoked potentials during microvascular decompression for hemifacial spasm. J Neurosurg 125(5): 1061-1067.

7. Bian X, Zhou R, Yang Y, Li P, Hang Y, et al. (2015) Divergent Effect of Dezocine, Morphine and
Sufentanil on Intestinal Motor Function in Rats. Int J Med Sci 12(11): 848-852.

8. Lee MH, Jee TK, Lee JA, Park K (2016) Postoperative complications of microvascular decompression for hemifacial spasm: lessons from experience of 2040 cases. Neurosurg Rev39(1): 151-158.

9. Hyun SJ, Kong DS, Park K (2010) Microvascular decompression for treating hemifacial spasm: lessons learned from a prospective study of 1,174 operations. Neurosurg Rev 33(3): 325-334.

10. Lee LA, Caplan RA, Stephens LS, Posner KL, Terman GW, et al. (2015) Postoperative opioid-induced respiratory depression: a closed claims analysis. Anesthesiology 122(3): 659-665.

11. Cashman JN, Dolin SJ (2004) Respiratory and haemodynamic effects of acute postoperative pain management: evidence from published data. $\mathrm{Br} \mathrm{J}$ Anaesth 93(2): 212-223.

12. Gan TJ, Habib AS, Miller TE, White W, Apfelbaum JL (2014) Incidence, patient satisfaction, and perceptions of post-surgical pain: results from a US national survey. Curr Med Res Opin 30(1): 149-160.

13. Frampton JE (2016) Sublingual Sufentanil: A Review in Acute Postoperative Pain. Drugs 76(6): 719-729.

14. Minkowitz HS (2015) A review of sufentanil and the sufentanil sublingual tablet system for acute moderate to Severe pain. Pain Manag 5(4): 237-250.

15. Wang C, Li L, Shen B, Jiang H, Yuan L, et al. (2014) A multicenter randomized double-blind prospective study of the postoperative patient controlled intravenous analgesia effects of dezocine in elderly patients. Int J Clin Exp Med 7(3): 530-539.

16. Garimella V, Cellini C (2013) Postoperative pain control. Clin Colon Rectal Surg 26(3): 191-196.

17. Kehlet H, Jensen TS, Woolf CJ (2006) Persistent postsurgical pain: risk factors and prevention. Lancet 367(9522): 1618-1625.

18. Shi F, Xiao Y, Xiong W, Zhou Q, Yang P, et al. (2015) Effects of Fentanyl on Emergence Agitation in Children under Sevoflurane Anesthesia: MetaAnalysis of Randomized Controlled Trials. PLoS One 10(8): e0135244. 


\section{Anaesthesia \& Critical Care Medicine Journal}

19. Morgan D, Cook CD, Smith MA, Picker MJ (1999) An examination of the interactions between the antinociceptive effects of morphine and various muopioids: The role of intrinsic efficacy and stimulus intensity. Anesth Analg 88(2): 407-413.

20. Katz J, Clarke H, Seltzer Z (2011) Preventive analgesia: quo vadimus? Anesth Analg 113(5): 12421253.

21. Wu L, Dong YP, Sun L, et al. (2015) Dezocine Prevents Postoperative Hyperalgesia in Patients Undergoing Open Abdominal Surgery. J Cardiothorac Vasc Anesth 29: 950-954.

22. O'Brien JJ, Benfield P (1989) Dezocine. A preliminary review of its pharmacodynamic and pharmacokinetic properties, and therapeutic efficacy. Drugs 38(2): 226-248.

23. Pandit SK, Kothary SP, Pandit UA, Kunz NR (1985) Double-blind placebocontrolled comparison of dezocine and morphine for post-operative pain relief. Can Anaesth Soc J 32(6): 583-591.

24. Ma Y, Li YF, Wang QC, Wang B, Huang HT (2016) Neurosurgical treatment of glossopharyngeal neuralgia: analysis of 103 cases. J Neurosurg 124(4): 1088-1092.

25. Jannetta PJ, Abbasy M, Maroon JC, Ramos FM, Albin MS (1977) Etiology and definitive microsurgical treatment of hemifacial spasm Operative techniques and results in 47 patients. J Neurosurg 47(3): 321328.
26. Wan X, Tang X, Wang Y (2002) The properties and longitudinal experience of Chinese Type A botulinum toxin for the treatment of focal dystonia and hemifacial spasm. Chung-kuo i Hsueh k'oHsueh tsa Chih/Chin Acad Med Sci 18: 254-259.

27. Barker FG, Jannetta PJ, Bissonette DJ, Philip TS, Mark $\mathrm{VL}$, et al. (1995) Microvascular decompression for hemifacial spasm. J Neurosurg 82(2): 201-210.

28. Qi H, Zhang W, Zhang X, Zhao C et al. (2016) Microvascular Decompression Surgery for Hemifacial Spasm. J Craniofac Surg 27(1): 124-127.

29. Li D, Fu C, Xu D, Sun L, Yu W, et al. (2013) Remote peritentorial hemorrhage complicating supratentorial aneurysmal surgery: a report of three cases and literature review. Acta Neurochir 155(2): 271-276.

30. Li N, Zhao W, Pu C, Shen J (2007) Delayed hemorrhage following microvascular decompression Three case reports. Neurol Med Chir 47(4): 186-188.

31. Hanakita J, Kondo A (1988) Serious complications of microvascular decompression operations for trigeminal Neuralgia and hemifacial spasm.Neurosurgery 22(2): 348-352.

32. An J, Fang Q, Huang C, Qian X, Fan $T$, et al. (2011)Deeper total intravenous anesthesia reduced the incidence of early postoperative cognitive dysfunction after microvascular decompression for facial spasm. J Neurosurg Anesthesiol 23(1): 12-17.

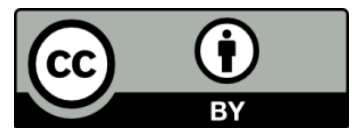

Ping Zhao, et al. Comparison of Cardiovascular, Analgesic and Sedative Effects of Different Doses of Dezocine. Anaesth Critic Care Med J 2017, 2(1): 000121. 